\title{
Hepatic Metabolomics Investigation in Acute and Chronic Murine Toxoplasmosis
}

\author{
Xiao-Qing Chen ${ }^{1,2}$, Hany M. Elsheikha ${ }^{3}$, Rui-Si Hu ${ }^{1}$, Gui-Xue $\mathrm{Hu}^{2}$, Shu-Ling Guo ${ }^{4}$, \\ Chun-Xue Zhou ${ }^{4 *}$ and Xing-Quan Zhu ${ }^{1 *}$ \\ ${ }^{1}$ State Key Laboratory of Veterinary Etiological Biology, Key Laboratory of Veterinary Parasitology of Gansu Province, \\ Lanzhou Veterinary Research Institute, Chinese Academy of Agricultural Sciences, Lanzhou, China, ${ }^{2}$ College of Animal \\ Science and Technology, Jilin Agricultural University, Changchun, China, ${ }^{3}$ Faculty of Medicine and Health Sciences, School \\ of Veterinary Medicine and Science, University of Nottingham, Loughborough, United Kingdom, ${ }^{4}$ Department of \\ Parasitology, Shandong University School of Basic Medicine, Jinan, China
}

OPEN ACCESS

Edited by:

Mario Alberto Rodriguez,

Centro de Investigación y de Estudios Avanzados del Instituto Politécnico Nacional (CINVESTAV-IPN), Mexico

Reviewed by:

Sandra Marcia Muxel, Universidade de São Paulo, Brazil Olgica Djurkovic-Djakovic, University of Belgrade, Serbia

*Correspondence: Chun-Xue Zhou zhouchunxue23@163.com Xing-Quan Zhu xingquanzhu1@hotmail.com

Received: 27 December 2017 Accepted: 17 May 2018 Published: 05 June 2018

Citation: Chen X-Q, Elsheikha HM, Hu R-S, Hu G-X, Guo S-L, Zhou C-X and

Zhu X-Q (2018) Hepatic Metabolomics Investigation in Acute and Chronic Murine Toxoplasmosis. Front. Cell. Infect. Microbiol. 8:189.

doi: 10.3389/fcimb.2018.00189
Toxoplasma gondii poses a great threat to human health, with no approved vaccine available for the treatment of T. gondii infection. T. gondii infections are not limited to the brain, and may also affect other organs especially the liver. Identification of host liver molecules or pathways involved in T. gondii replication process may lead to the discovery of novel anti-T. gondii targets. Here, we analyzed the metabolic profile of the liver of mice on 11 and 30 days postinfection (dpi) with type II T. gondii Pru strain. Global metabolomics using liquid chromatography-tandem mass spectrometry (LC-MS/MS) identified 389 significant metabolites from acutely infected mice; and 368 from chronically infected mice, when compared with control mice. Multivariate statistical analysis revealed distinct metabolic signatures from acutely infected, chronically infected and control mice. Infection influenced several metabolic processes, in particular those for lipids and amino acids. Metabolic pathways, such as steroid hormone biosynthesis, primary bile acid biosynthesis, bile secretion, and biosynthesis of unsaturated fatty acids were perturbed during the whole infection process, particularly during the acute stage of infection. The present results provide insight into hepatic metabolic changes that occur in BALB/c mice during acute and chronic T. gondii infection.

Keywords: toxoplasmosis, host-pathogen interaction, liver, non-targeted metabolomics, LC-MS/MS

\section{INTRODUCTION}

Toxoplasma gondii infection poses a serious health problem worldwide (Dubey, 2008; Elsheikha, 2008; Dupont et al., 2012). This parasite has a complex life cycle that involves both sexual and asexual phases. Sexual reproduction results in the formation and shedding of the oocysts by the felid definitive host (Sullivan and Jeffers, 2012). Ingestion of food or drinking water contaminated with oocysts is one of the most important sources of infection (Dubey and Beattie, 1988). Asexual reproduction results in the formation of tachyzoites and bradyzoites-containing tissue cysts, marking acute and chronic infection, respectively (Munoz et al., 2011; Sullivan and Jeffers, 2012). The transformation of tachyzoites into tissue cysts is immune-mediated and leads to the establishment of latent infection (Dubey and Frenkel, 1976). Ingesting undercooked or raw meat that contains tissue cysts is another important source of infection (Dubey and Beattie, 1988). Although the brain and spinal cord are the primarly affected organs (Elsheikha and Zhu, 2016), 
T. gondii infections are not limited to the central nervous system, and may also affect other organs. Several hepatic abnormalities, including hepatomegaly, hepatitis, granuloma, necrosis, cholestatic jaundice, cirrosis, and abnormalities in liver enzymes have been observed in the context of T. gondii infection (Vischer et al., 1967; Weitberg et al., 1979; Wendum et al., 2002). However, the definite pathophysiological links between $T$. gondii and extraneural manifestations are yet to be established.

The association between $T$. gondii infection and hepatic injury has attracted considerable attention due to the significant roles played by the liver in maintaining many body functions. The mechanisms leading to hepato-pathologic disturbances in $T$. gondii-infected mice have been investigated using transcriptomics (He et al., 2016a) and proteomics ( $\mathrm{He}$ et al., 2016b). However, the mechanisms underlying the hepatic pathological outcomes of $T$. gondii infection remain unuclear. T. gondii, a strict intracellular parasite, relies on host cells to obtain its energetic and nutritional needs (Kafsack and Llinas, 2010). The parasite utilizes essential nutrients from the host cell, including tryptophan (Pfefferkorn, 1984; Dai et al., 1994; Naemat et al., 2016); arginine (Fox et al., 2004); polyamines (Seabra et al., 2004); purines (Schwartzman and Pfefferkorn, 1982; Chaudhary et al., 2004); cholesterol (Coppens et al., 2000; Coppens and Joiner, 2003) and other nutrients (Dimier and Bout, 1998; Charron and Sibley, 2002). Hence, it is reasonable to assume that infected cells display different metabolic phenotypes, and that the metabolomic alterations and associated dysregulation of biochemical pathways may underlie the hepatopathy observed in toxoplasmosis.

In recent years, transcriptomics and proteomics approaches have been employed to study the interaction between $T$. gondii and its host (Nelson et al., 2008; Pittman et al., 2014; He et al., 2016a,b; Cong et al., 2017). Metabolomics is a rapidly emerging field of "omics" research and can provide a global analysis of the metabolic changes in biological systems in response to external stimuli or perturbation (Nicholson et al., 1999; Yang et al., 2006; Holmes et al., 2008). Metabolomics has been as a powerful method to profile metabolic alterations associated with microbial infections (Antunes et al., 2011; Shin et al., 2011; Nguyen et al., 2015). In previous studies, we characterized the metabolomic profiles of the brain (Zhou et al., 2015), serum (Zhou et al., 2016), and spleen (Chen et al., 2017) of mice infected with $T$. gondii. In this study, we extended our metabolomics analysis to include the liver due to its important role in maintaining many metabolic processes in the body. Here, the liver metabolic profile was compared between mice with acute infection and mice with chronic infection using liquid chromatography-tandem mass spectrometry (LC-MS/MS). This approach revealed metabolic differences between infected and uninfected mice, and between acutely infected and chronically infected mice.

\section{MATERIALS AND METHODS}

\section{Ethical Approval}

All experiments were approved by the Animal Administration and Ethics Committee of Lanzhou Veterinary Research Institute, Chinese Academy of Agricultural Sciences (Permit No.
LVRIAEC2016-007). Mice were handled in strict accordance with good laboratory animal practice according to the Animal Ethics Procedures and Guidelines of the People's Republic of China. All efforts were made to minimize animal suffering and to minimize the number of animals used in the experiments.

\section{Mice Infection}

Thirty-nine, 6-week-old, female, BALB/c mice were obtained from the Laboratory Animal Center of Lanzhou Veterinary Research Institute, Chinese Academy of Agricultural Sciences. Mice were housed in microisolator cages under specific-pathogen free (SPF) conditions, with controlled temperature $\left(22 \pm 2^{\circ} \mathrm{C}\right)$ and $12 \mathrm{~h}$ light/dark cycles, and were given water and standard food pellets ad libitum. Mice were randomly allocated into three groups: acutely infected group $(n=13)$, chronically infected group $(n=13)$ and healthy uninfected group $(n=13)$. Each mouse in the infected group was infected orally with 10 tissue cysts of T. gondii type II Pru strain that were isolated from mice brain 40 days postinfection (dpi) in $100 \mu l$ phosphate buffered saline solution (PBS). Control mice were gavaged with $100 \mu \mathrm{l}$ PBS only. All mice were observed daily for the development of clinical signs and for survival throughout the entire experiment. Also, weight loss was monitored as a sign of morbidity.

\section{Collection of Tissues and Characterization of Infection}

At 11 days postinfection (dpi), 10 mice from the acutely infected group were sacrificed and their livers were collected. A further 10 mice representing chronically infected group, and 10 uninfected controls, were sacrificed and their livers were collected at 30 dpi. Mice were anesthetized by isoflurane inhalation and then sacrificed by exsanguination via cardiac puncture. Livers were rapidly removed, cut into small pieces, rinsed with saline solution $(0.9 \% \mathrm{NaCl} \mathrm{w} / \mathrm{v})$, snap-frozen in liquid nitrogen, and stored at $-80^{\circ} \mathrm{C}$. Mice brain collected at $30 \mathrm{dpi}$ were checked for the presence of $T$. gondii cysts using a light microscope. Other organs (including heart, lung, muscle, small intestine and kidney) were examined for the presence of $T$. gondii, as previously described (Hill et al., 2006). Briefly, total genomic DNA was extracted from these various tissues using QIAamp ${ }^{\circledR}$ DNA Mini kit in accordance with the manufacturer's instructions (QIAGEN, Germany). Isolated DNA was then used as a template for PCR amplification of the T. gondii $\mathrm{B} 1$ gene using the specific primers (5'-AACGGG CGA GTA GCA CCT GAG GAG-3' and $5^{\prime}$-TGG GTC TAC GTC GAT GGC ATG ACA AC-3'). Positive control (DNA from T. gondii) and negative (deionized water) control samples were included in each PCR run. Sections of livers from 9 mice ( 3 from each of the acutely infected, chronically infected, and control groups) were collected and processed for routine histopathological examination. Tissues were fixed by submersion in $10 \%$ neutral buffered formalin for 1 week, then dehydrated in a graded series of ethanol and embedded in paraffin wax. Sections were made $(5 \mu \mathrm{m})$ using a microtome, then stained with hematoxylin-eosin (H\&E) and examined under an optical microscope (Olympus, Tokyo, Japan). 


\section{Extraction of Metabolites}

Liver samples were taken from storage in a $-80^{\circ} \mathrm{C}$ freezer and allowed to thaw at $4^{\circ} \mathrm{C}$. The organic protein precipitation method was used to extract metabolites. Briefly, $25 \mathrm{mg}$ tissue samples were weighed and then pulverized in liquid nitrogen. Then, $800 \mu \mathrm{l}$ methanol/water (1:1) solution and $3 \mathrm{~mm}$ (mean diameter) steel beads were added to each sample. Using a TissueLyser bead-mill homogenizer (QIAGEN, Hilden, Germany), samples were homogenized via vibrating at $60 \mathrm{~Hz}$ for $5 \mathrm{~min}$. Subsequently, samples were centrifuged at $25,000 \mathrm{~g}$ for $10 \mathrm{~min}$ at $4^{\circ} \mathrm{C}$. To evaluate the reproducibility and stability of the LC-MS/MS system, $200 \mu \mathrm{l}$ supernatant of each sample was mixed to generate a pooled quality control (QC) sample. The supernatant from each sample was separated and freeze dried. Finally, dried supernatant samples were reconstituted in deionized water and analyzed by mass spectrometry.

\section{LC-MS/MS Analysis}

An ultra-performance liquid chromatography (UPLC) system (Waters, USA) was used to perform all analyses. The Chromatographic separation was achieved using an ACQUITY UPLC BEH C18 column $(100 \mathrm{~mm} * 2.1 \mathrm{~mm}, 1.7 \mu \mathrm{m}$, Waters, USA) at a column temperature of $50^{\circ} \mathrm{C}$ and flow rate of $0.4 \mathrm{ml} / \mathrm{min}$, wherein the mobile phase consisted of solvent $\mathrm{A}$ (water $+0.1 \%$ formic acid) and solvent $\mathrm{B}$ (acetonitrile + $0.1 \%$ formic acid). The following gradient elution conditions were used to elute metabolites: $100 \%$ phase A for $0-2 \mathrm{~min} ; 0 \%$ to $100 \%$ phase $\mathrm{B}$ for $\sim 11 \mathrm{~min} ; 100 \%$ phase $\mathrm{B}$ for $11-13 \mathrm{~min}$; $100 \%$ phase A for $13-15 \mathrm{~min}$. Injection volume per sample was $10 \mu \mathrm{l}$. The metabolites eluted from the column were detected using a high-resolution tandem mass spectrometer SYNAPT G2 XS QTOF (Waters, USA) in positive and negative electrospray ionization modes. For the positive ionization mode, the capillary voltage and the cone voltage were set at $2 \mathrm{kV}$ and $40 \mathrm{~V}$, respectively. For the negative ionization mode, these parameters were set at $1 \mathrm{kV}$ and $40 \mathrm{~V}$, respectively. Centroid MSE mode was used to obtain the mass spectrometry data. The primary scan ranged from $50 \mathrm{Da}$ to $1200 \mathrm{Da}$ with a scanning time of $0.2 \mathrm{~s}$. All the parent ionizations were fragmented at $20-40 \mathrm{~V}$ and the information from the fragments was collected. During data acquisition, the LE signal was gained every $3 \mathrm{~s}$ for real-time quality correction. Furthermore, quality control samples (10 samples) were collected in order to evaluate the stability of the instrument during measurements.

\section{Data Processing and Statistical Analysis}

UPLC-MS/MS raw data files were imported into Progenesis QI software (Waters, UK). Data were processed for possible adducts, peak alignment, peak detection, deconvolution, dataset filtering, noise reduction, compound identification, and normalization with sum method. Chromatograms were aligned to a reference QC run and selected adducts included $[\mathrm{M}+\mathrm{H}]^{+},[\mathrm{M}+\mathrm{Na}]^{+}$, $[\mathrm{M}+\mathrm{K}]^{+},[\mathrm{M}+\mathrm{NH} 4]^{+}$for $\mathrm{ESI}^{+}$and $[\mathrm{M}-\mathrm{H}]^{-},[\mathrm{M}+\mathrm{Cl}]^{-}$for ESI- with $2.5 \mathrm{AU}$ filter. Peak picking was set to exclude ions eluted before $0.5 \mathrm{~min}$ and after $9 \mathrm{~min}$ at $1 \%$ and $92 \%$ organic solution, respectively. The raw data were normalized using a global scaling factor to align all injections to a reference run by adjusting for the quantitative abundance of each detectable feature, correcting for experimental variation based on deviation from the median. The results were exported in.CSV format for further analysis and reporting of selected ions by their accurate mass and retention time pair. The normalized peak data were further processed by an in-house software metaX (Wen et al., 2017). Those features that were detected in less than $50 \%$ of QC samples or $80 \%$ of biological samples were removed, and the remaining peaks with missing values were imputed with the k-nearest neighbor algorithm to further improve the data quality. Then, the ions that demonstrated relative standard deviation (RSD) greater than $30 \%$ in QC pools were filtered out.

Multivariate statistical analyses including Principal Component Analysis (PCA) and Partial Least SquaresDiscriminant Analysis (PLS-DA) were performed to discriminate infected mice from control mice. PCA was performed for outlier detection and batch effects evaluation using the pre-processed dataset. Supervised PLS-DA was conducted through metaX to discriminate the different variables between groups. The condition of $p$-values obtained from a two-tailed, Student's $t$-test on the normalized peak areas ( $q$-values $) \leq 0.05$ and Fold Change $(\mathrm{FC}) \geq 1.2$ or $\leq 0.833$, were taken into consideration during the selection of candidate metabolites. $\log 2$ FC based on metabolite abundance was used to demonstrate how the differential abundance of liver metabolites varied between the mouse groups. Differential metabolites were identified based on $q$-values (adjusted p-values using Benjamini-Hochberg procedure) and false discovery rate $(\mathrm{FDR})$ of $\leq 0.05, \mathrm{FC} \geq 1.2$ or $\leq 0.833$, and variable importance in projection (VIP) score $\geq 1$ from the PLS-DA model. Data were log2-transformed prior to cluster analysis and the generation of heat-maps using $\mathrm{R}$, to show distinctions in the metabolic state between infected mice and controls, and between infected mice at different stages of infection.

\section{Identification of Metabolites}

Metabolite identification was performed by matching the exact molecular mass data $(\mathrm{m} / z)$ of samples with the online KEGG (www.genome.jp/kegg/) database. A mass error range of $10 \mathrm{ppm}$ was set to match the compound and obtain a mass accuracy score (Zhong et al., 2017). Progenesis QI software was used to match the theoretical isotope distribution in the database, and obtain the isotopic similarity score of the metabolites. Then, all the possible compounds corresponding to each parent ion in the first-order spectrum were theoretically fragmented according to their molecular structure to obtain the theoretical fragment ions. The spectra of all theoretical fragment ions together formed the theoretical secondary spectrum. Then, we set the mass error range of $10 \mathrm{ppm}$, and matched the actual secondary spectrum of the experimental samples with the theoretical secondary spectrum. Scores of the matching similarity between the spectra were counted. Finally, the three scores were combined into total assessment scores, which were used to screen the metabolites. 


\section{RESULTS}

\section{Clinical and Histopathological Analysis}

Infected mice exhibited a time-dependent increase in progression of disease. At $7 \mathrm{dpi}$, infected mice developed mild loss of appetite and ruffled hair coat. On day 11 postinfection, clinical signs had progressed to those typically observed during acute infection, including anorexia, weight loss, edema and ruffled hair. By day 14 postinfection infected mice began to recover from acute signs, and by day 30 postinfection all infected mice had restored their normal physical state and developed chronic infection. As expected, control mice did not show any clinical manifestations throughout the experiment. PCR results of all examined tissues indicated that all infected mice tested positive, but control mice tested negative. A histopathological study of liver tissue from all mice was also performed, in order to ascertain both acute (11 dpi) and chronic (30 dpi) histopathologies. Notable histological changes were observed in acutely infected mice, which showed multifocal mononuclear cell aggregations with numerous vacuolated hepatocytes (Figure 1A). These changes featured less prominently in chronically infected mice (Figure 1B). Livers of the control mice showed no gross histopathological abnormalities (Figure 1C). The original image of Figures 1A-C are Figures S1-S3, respectively.

\section{Metabolite Profiling of Liver During T. gondii Infection}

To investigate the hepatic metabolic profiles during infection progression, liver extracts of $T$. gondii-infected mice and uninfected mice were analyzed with an UPLC-MS/MS system. Analysis of the total ion chromatograms (TIC) and PCA score plots of QC samples demonstrated the high stability, consistency and reproducibility of chromatographic separation as shown in Figure S4A (ESI+) and Figure S4B (ESI-). The PCA scores plot showed that the uninfected mice cluster distinctly to the right; whereas the infected mice (acutely infected and chronically infected) cluster slightly to the left without overlapping with the uninfected mice and QC samples cluster together as shown in Figure 2A (ESI+) and Figure 2B (ESI-). We also performed PLS-DA analysis to compare the three different mouse groups, including the uninfected and infected (acutely infected and chronically infected) mice, as shown in Figure 2C (ESI+) and Figure 2D (ESI-). The PLS-DA analysis showed a high degree of segregation between the various mouse groups in the positive and negative electrospray ionization modes, further supporting the reproducibility of the data.

\section{Differential Metabolites During Different Infection Phases}

Differential metabolites were identified on the basis of VIP $\geq 1$ in the PLS-DA model and having a $q$-value $<0.05$ or a fold-change $\geq 1.2$ with high statistical significance (as shown in Table S1); including differential ion number, and the identified up-regulated and down-regulated ions between different experimental groups. In Table S1, we show that liver metabolic profile in acutely infected mice had the largest fold change, when compared with controls. As the infection progressed, the number of differential metabolites was relatively decreased. Meanwhile, the concentration of differential metabolites from acutely infected mice changed more significantly than those from chronically infected mice. These differential concentrations of metabolites from distinct groups were used for cluster analysis and generation of heatmaps, as shown in Figure 3A (ESI+) and Figure 3B (ESI). The heat-maps showed distinct differences between the infected and the control mice, as well as between acutely infected and chronically infected mice. We identified 389 significant metabolites from acutely infected group vs. control (Table S2) and 368 from the chronically infected group vs. control (Table S3). A Venn diagram (Figure 4) shows the correlation between metabolites according to the infection phases, with 205 differential metabolites overlapping between the two infection phases. KEGG analysis identified 51 differential metabolites from both infection phases linked to metabolic pathways (Table 1). Further, we randomly identified several differential metabolites from Table 1, including anandamide, cholesterol, choli acid, lithocholic acid, L-Thyroxine, oleic acid and sphinganine (Figures S5-S11), using standard reference substances obtained from Sigma-Aldrich (St. Louis, USA).

\section{Perturbed Metabolic Pathways During Different Infection Phases}

According to the KEGG database annotations, there were 85 differential metabolites identified from the acutely infected group vs. control group and 92 differential metabolites from chronically infected vs. control groups, involved in metabolic pathways. The most significantly perturbed metabolic pathways and the number of upregulated and downregulated differentially abundant metabolites in each perturbed metabolic pathway, are shown (Figure 5). In the acutely infected phase: the six most perturbed metabolic pathways were involved in steroid hormone biosynthesis, primary bile acid biosynthesis, bile secretion, neuroactive ligand-receptor interaction, ubiquinone and other terpenoid-quinone biosynthesis, and the biosynthesis of unsaturated fatty acids (Figure 5A). In the chronically infected phase: steroid hormone biosynthesis, primary bile acid biosynthesis, bile secretion, neuroactive ligand-receptor interaction, biosynthesis of unsaturated fatty acids, and arachidonic acid (AA) metabolism were the six most perturbed metabolic pathways (Figure 5B). More metabolites were perturbed in AA metabolism in the chronically infected phase, when compared to the acutely infected phase. In the acutely infected phase, Leukotriene C4 was upregulated, whereas 20-COOH-LB4 and 11,12-DHET involved in AA metabolic pathway were downregulated. In the chronically infected phase, Leukotriene C4, 20-COOH-LB4, Prostaglandin E2, Prostacyctin and 15-OxoETE involved in AA metabolism were upregulated and only 11,12-DHET was downregulated. These results indicate that AA metabolism was downregulated during the acutely infected phase, but upregulated in chronic infection. A schematic illustration of the AA metabolic pathway is provided to show specific metabolites dysregulated during each infection phase (Figure 6). Multiple differentially abundant metabolites 


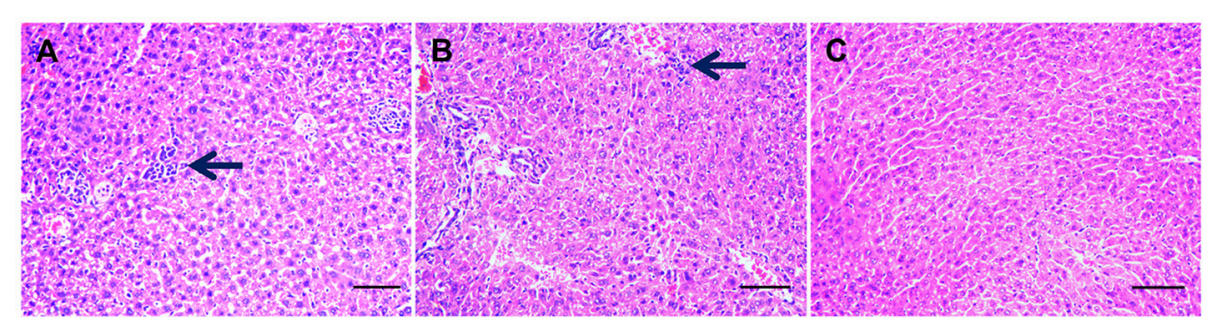

FIGURE 1 | Histopathological changes in the liver tissues from Toxoplasma gondii-infected mice. Histopathological analysis was conducted on livers from mock- and T. gondii-infected mice at days 11 and 30 postinfection. Photomicrographs of liver tissue sections stained with hematoxylin and eosin (original magnification, $\times 100$ ). (A) Liver section from a mouse on day 11 postinfection. Liver shows multifocal mononuclear cell aggregations (mainly lymphocytes, arrow) with numerous vacuolated hepatocyte. (B) At 30 dpi, liver shows mild mononuclear cellular infiltration (arrow), increased numbers of Von Kupffer cells besides numerous pyknotic hepatocytes. (C) Hepatic tissue from mock-infected, control mice shows a normal histological structure.
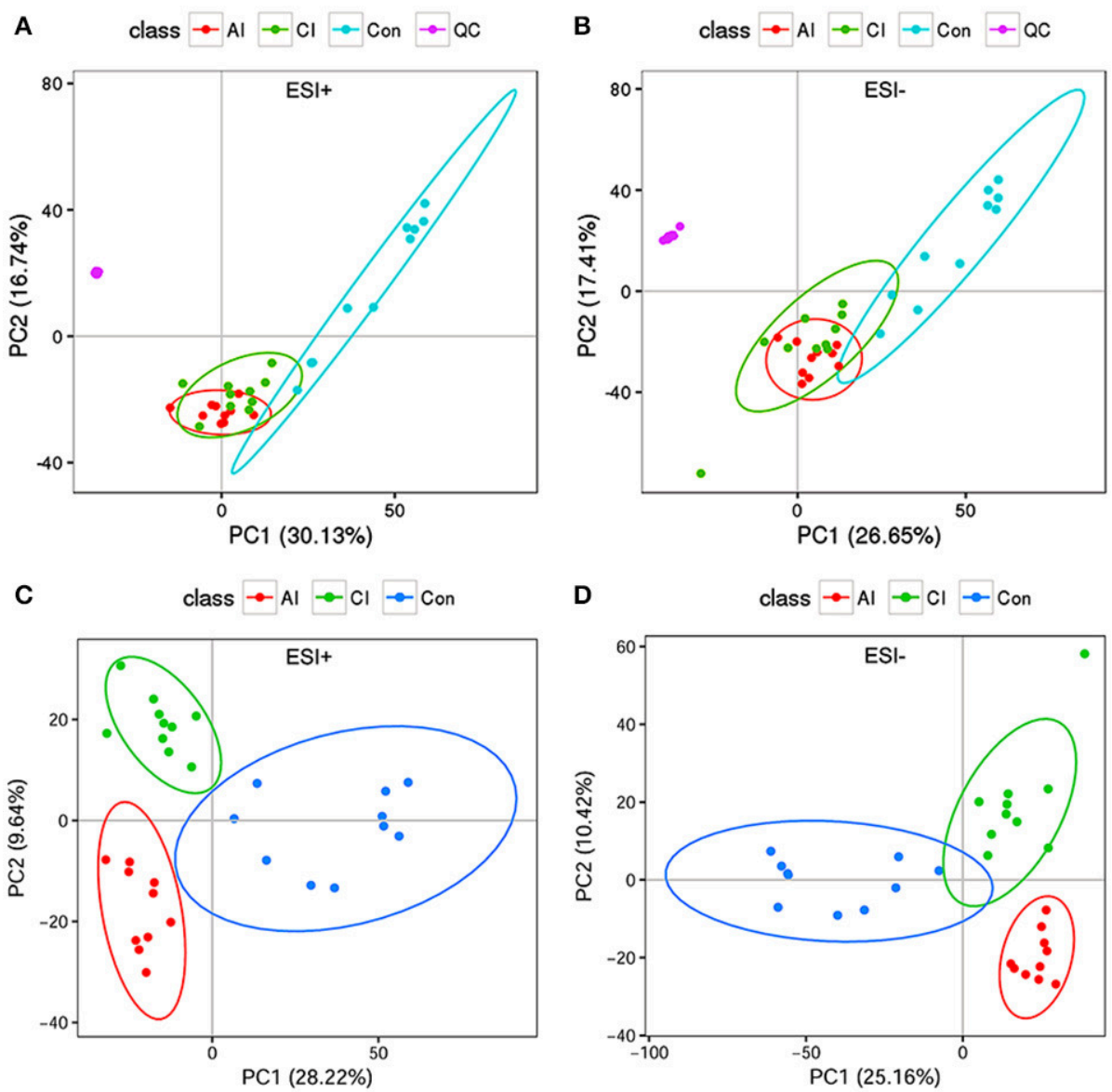

FIGURE 2 | Multivariate statistical analysis of the data. (A) PCA scores plot of mice livers, including acutely infected (Al), chronically infected (CI) and uninfected control (Con) compared to quality control (QC) samples in the positive ion mode (ESI+). (B) PCA scores plot of mice livers in the negative ion mode (ESI-). Clear separation was detected among the different mice groups and in relation to QC samples. (C) Two dimensional PLS-DA score plots of the acutely infected (Al), chronically infected $(\mathrm{Cl})$ and uninfected control (Con) in the positive ion mode (ESI+). (D) Two dimensional PLS-DA score plots of Al, Cl and Con in the negative ion mode (ESI-). Each dot represents one liver sample, projected onto first (horizontal axis) and second (vertical axis) PLS-DA variables. Mice groups are shown in different colors. The black ellipse indicates the $95 \%$ confidence interval.

involved in amino acid metabolism were also observed (Table 2), indicating that amino acid metabolism is significantly altered during T. gondii infection. Multiple amino acid metabolic pathways, such as tyrosine metabolism, arginine and proline metabolism, tryptophan metabolism, histidine metabolism, glycine, serine and threonine metabolism, cysteine and 


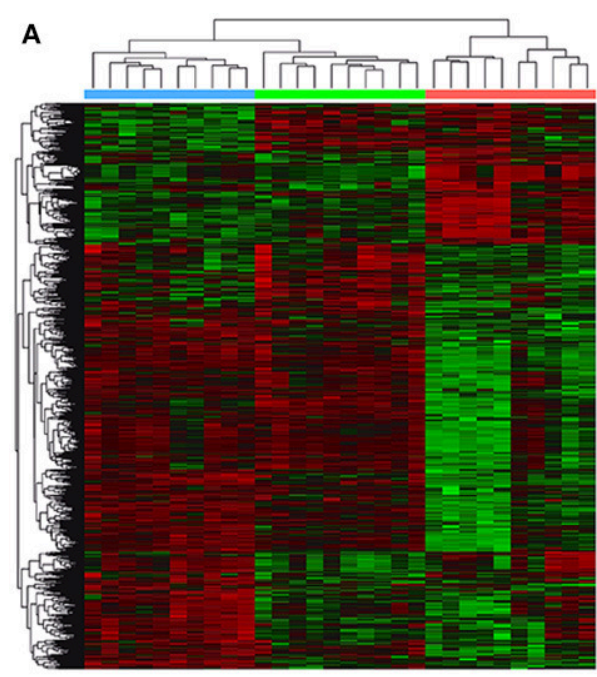

ESI+

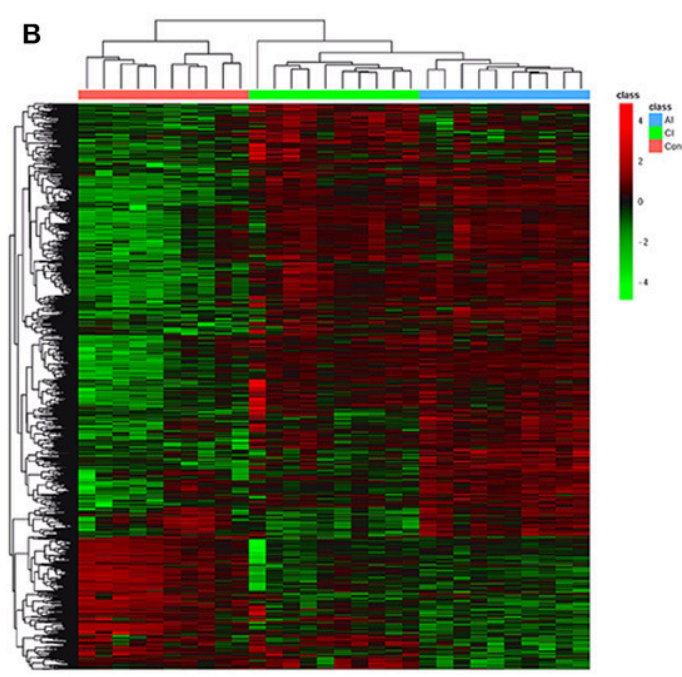

ESI-

FIGURE 3 | (A) Heatmaps of the differential metabolites in acutely infected mice (Al) and chronically infected mice (Cl) vs. control mice (Con) in the positive ion mode (ESI+). (B) Heatmaps of the differential metabolites in acutely infected mice (Al) and chronically infected mice (Cl) vs. control mice (Con) in the negative ion mode (ESI-). Red and green colors indicate values above and below the mean, respectively. Black indicates values close to the mean.

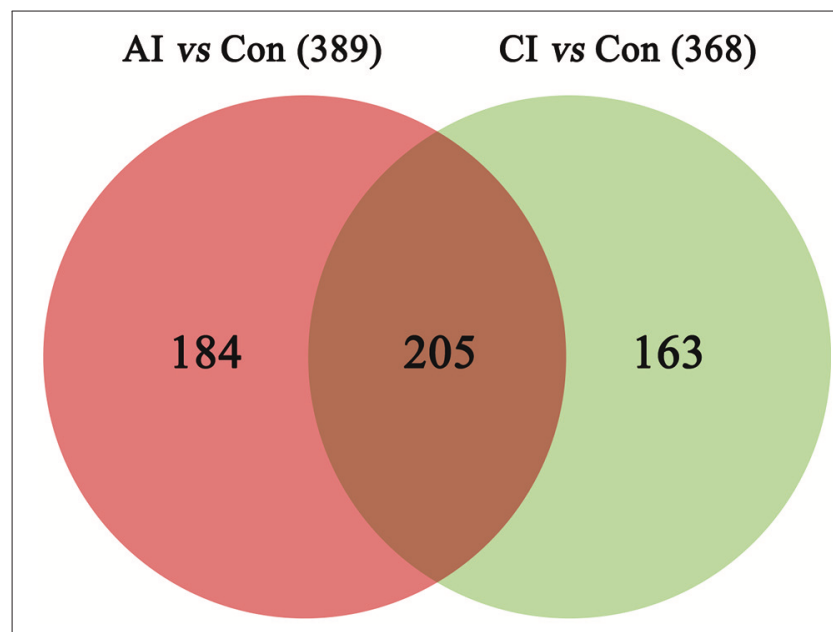

FIGURE 4 | A Venn diagram showing the common and unique metabolites between the acutely and chronically infected mice vs. control. In total, 389 metabolites were found during acute infection vs. control (red); and 368 metabolites were identified in chronically infected mice vs. control (green), 205 of which were shared between the two groups.

methionine metabolism, taurine and hypotaurine metabolism and glutathione metabolism, were altered in the infection groups.

\section{DISCUSSION}

A non-targeted metabolomics-based approach is presented that enabled the study of liver metabolites and metabolic pathways during T. gondii infection with the aim of defining infection-specific and stage-specific metabolic signatures.
This approach revealed 389 and 368 significantly altered metabolites in acutely infected mice and chronically infected mice, respectively, vs. control. The decreased number of altered metabolites during late infection indicates the restoration of the metabolic state. 205 differential metabolites were common to both acutely and chronically infected mice, of these 51 differential metabolites were involved in metabolic pathways (Table 1).

Previous LC-MS metabolomics analysis of mouse brain detected 60 differentially abundant metabolites at different stages of T. gondii infection (Zhou et al., 2015). Liquid chromatography-quadrupole time-of-flight mass spectrometry (LC-Q-TOF-MS)-based metabolomics analysis identified 79 and 74 differentially abundant metabolites in ESI+ mode and ESI- mode, respectively, in sera of T. gondiiinfected mice compared to controls (Zhou et al., 2016). LC-MS/MS-based metabolomics analysis of T. gondiiinfected mouse spleen revealed 132 differentially abundant metabolites (23 metabolites from acutely infected mice and 109 metabolites from chronically infected mice) compared with control (Chen et al., 2017). This discord between metabolic phenotyping of $T$. gondii infected tissues can be attributed to heterogeneity in host tissue types or to variation in the experimental conditions and the analytical protocols used in these studies.

The liver response to infection should be strong enough to control the parasite and in the meantime restrained in order to minimize immune-inflammatory pathology. Achieving this balance between protection against infection and immunopathology-induced tissue damage is essential in order to limit liver injury during T. gondii infection. We identified a number of altered metabolites and metabolic pathways involved 
TABLE 1 | List of the differential metabolites involved in the perturbed metabolic pathways during acute and chronic phases of Toxoplasma gondii infection.

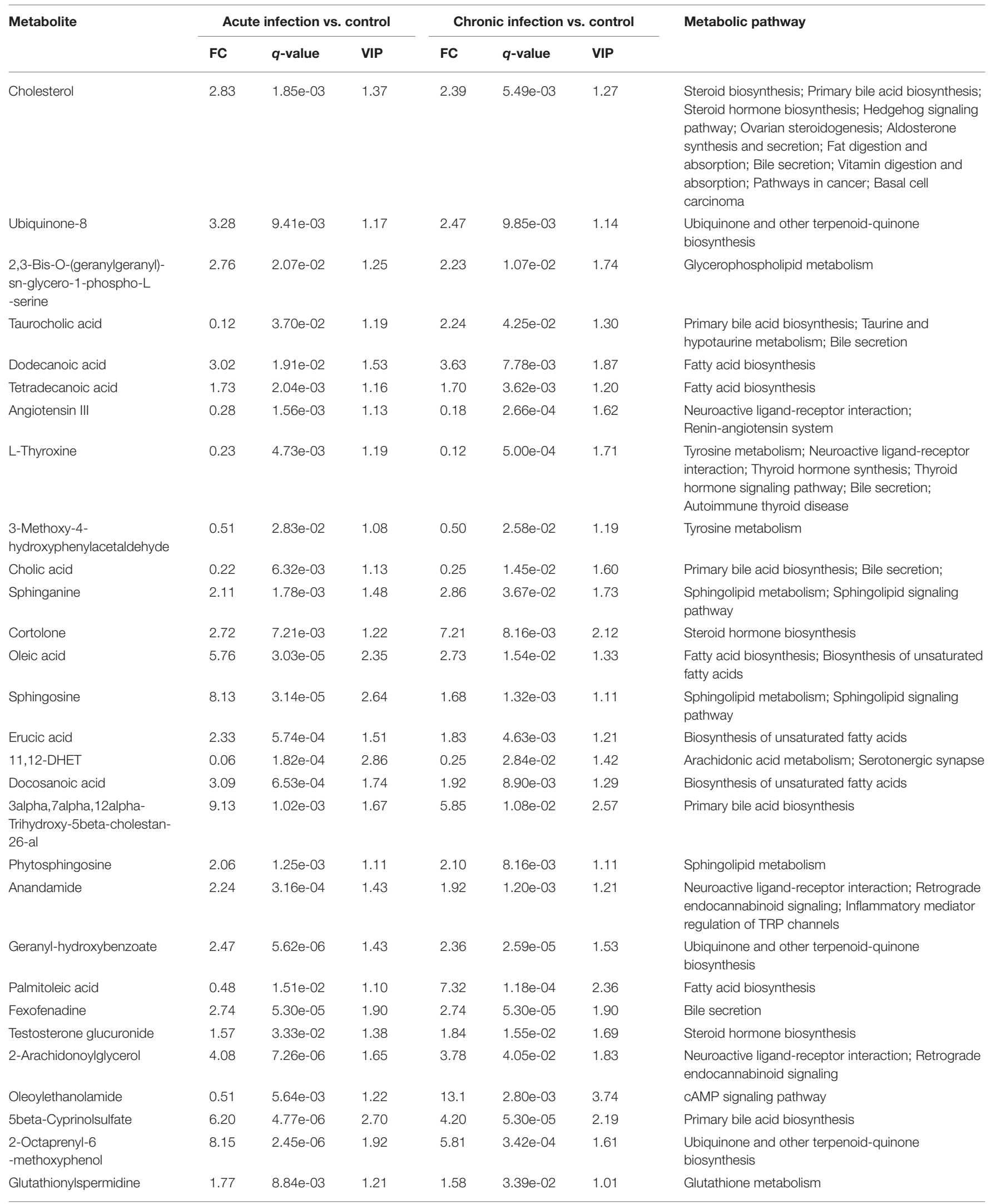


TABLE 1 | Continued

\begin{tabular}{|c|c|c|c|c|c|c|c|}
\hline \multirow[t]{2}{*}{ Metabolite } & \multicolumn{3}{|c|}{ Acute infection vs. control } & \multicolumn{3}{|c|}{ Chronic infection vs. control } & \multirow[t]{2}{*}{ Metabolic pathway } \\
\hline & FC & $q$-value & VIP & FC & $q$-value & VIP & \\
\hline Vitamin D3 & 2.53 & $2.20 \mathrm{e}-03$ & 1.26 & 2.59 & $8.83 e-03$ & 1.33 & $\begin{array}{l}\text { Steroid biosynthesis; Vitamin digestion and } \\
\text { absorption; Rheumatoid arthritis }\end{array}$ \\
\hline $\begin{array}{l}\text { 7alpha-Hydroxycholest-4 } \\
\text {-en-3-one }\end{array}$ & 2.03 & $6.38 \mathrm{e}-04$ & 1.13 & 3.35 & $5.75 e-03$ & 2.13 & Primary bile acid biosynthesis \\
\hline Lithocholic acid & 2.06 & $4.08 \mathrm{e}-02$ & 1.40 & 0.67 & $1.88 e-04$ & 1.01 & Bile secretion \\
\hline 20-Hydroxycholesterol & 2.31 & $2.52 \mathrm{e}-03$ & 1.21 & 5.90 & $5.44 e-05$ & 2.42 & Steroid hormone biosynthesis \\
\hline 7-Dehydrodesmosterol & 2.79 & $4.07 e-05$ & 1.68 & 2.00 & $2.49 e-03$ & 1.21 & Steroid biosynthesis \\
\hline 7alpha-Hydroxycholesterol & 4.72 & $3.78 e-03$ & 1.90 & 2.06 & $8.52 e-03$ & 1.26 & Primary bile acid biosynthesis \\
\hline Nervonic acid & 11.1 & $6.37 e-05$ & 2.98 & 6.25 & $5.38 e-04$ & 2.38 & Biosynthesis of unsaturated fatty acids \\
\hline Bradykinin & 1.94 & $1.95 \mathrm{e}-02$ & 1.62 & 1.94 & $2.95 e-02$ & 1.59 & $\begin{array}{l}\text { cGMP-PKG signaling pathway; Sphingolipid } \\
\text { signaling pathway; Neuroactive ligand-receptor } \\
\text { interaction; Complement and coagulation cascades; } \\
\text { Inflammatory mediator regulation of TRP channels; } \\
\text { Regulation of actin cytoskeleton; Chagas disease } \\
\text { (American trypanosomiasis); African } \\
\text { trypanosomiasis; Pathways in cancer }\end{array}$ \\
\hline 20-COOH-LTB4 & 0.37 & $1.49 \mathrm{e}-02$ & 1.60 & 1.90 & $1.78 e-02$ & 1.01 & Arachidonic acid metabolism \\
\hline $\begin{array}{l}\text { 3alpha, } 11 \text { beta,21- } \\
\text { Trihydroxy-20-oxo-5beta- } \\
\text { pregnan-18-al }\end{array}$ & 0.33 & $3.39 e-04$ & 1.70 & 2.65 & $9.53 e-03$ & 1.36 & Steroid hormone biosynthesis \\
\hline Leukotriene C4 & 3.14 & $1.46 \mathrm{e}-02$ & 2.05 & 3.03 & $2.19 e-02$ & 2.09 & $\begin{array}{l}\text { Arachidonic acid metabolism; Neuroactive } \\
\text { ligand-receptor interaction; Fc epsilon RI signaling } \\
\text { pathway; Serotonergic synapse; Bile secretion; } \\
\text { Asthma }\end{array}$ \\
\hline Delta-Tocotrienol & 4.71 & $2.65 e-04$ & 1.64 & 5.17 & $4.88 e-03$ & 1.58 & $\begin{array}{l}\text { Ubiquinone and other terpenoid-quinone } \\
\text { biosynthesis }\end{array}$ \\
\hline Trypanothione disulfide & 2.16 & $8.94 \mathrm{e}-03$ & 1.15 & 2.66 & $3.34 \mathrm{e}-03$ & 1.56 & Glutathione metabolism \\
\hline Taurolithocholate sulfate & 4.44 & $1.43 e-04$ & 2.17 & 5.28 & $1.52 \mathrm{e}-04$ & 2.49 & Bile secretion \\
\hline Icosadienoic acid & 6.03 & $1.86 e-04$ & 1.80 & 4.26 & $4.24 \mathrm{e}-03$ & 1.37 & Biosynthesis of unsaturated fatty acids \\
\hline 5"-Phosphoribostamycin & 0.18 & $1.00 \mathrm{e}-05$ & 2.24 & 0.32 & $6.07 e-04$ & 1.54 & Butirosin and neomycin biosynthesis \\
\hline Trypanothione & 9.45 & $1.81 \mathrm{e}-03$ & 3.28 & 6.21 & $5.32 e-03$ & 2.95 & Glutathione metabolism \\
\hline Docosapentaenoic acid & 2.70 & $1.87 e-05$ & 1.30 & 2.14 & $1.22 \mathrm{e}-03$ & 1.02 & Biosynthesis of unsaturated fatty acids \\
\hline Pregnanolone & 2.78 & $5.62 e-03$ & 1.16 & 2.27 & $1.77 e-03$ & 1.15 & Steroid hormone biosynthesis \\
\hline Phospho-anandamide & 2.20 & $5.41 e-05$ & 1.12 & 2.29 & $1.09 e-04$ & 1.28 & Retrograde endocannabinoid signaling \\
\hline 13,16-Docosadienoic acid & 9.59 & $1.25 e-04$ & 2.58 & 4.40 & $1.74 \mathrm{e}-03$ & 1.83 & Biosynthesis of unsaturated fatty acids \\
\hline Calcitetrol & 18.3 & $1.75 e-03$ & 3.62 & 4.98 & $3.22 \mathrm{e}-02$ & 2.43 & Steroid biosynthesis \\
\hline
\end{tabular}

FC, fold change; $q$-value, adjusted $p$ value calculated by two-tailed Wilcoxon rank-sum tests after false discovery rate correction; VIP, variable importance in projection.

in the inflammatory response to T. gondii infection. Taurine, the most abundant free amino acid in the liver and other tissues, has antioxidant and anti-inflammatory activities (Rosa et al., 2014). Reduced level of taurocholic acid that is involved in bile acid biosynthesis, taurine and hypotaurine metabolism, was detected during acute infection, probably to facilitate the parasite establishment. This taurine-related reduction in the anti-inflammatory status seemed to be buffered by increased levels of glutathionylspermidine, trypanothione disulfide and trypanothione metabolites involved in glutathione (GSH) metabolism during acute and chronic infections (Silva et al., 2002; Marino and Boothroyd, 2017). GSH is an important antioxidant that plays an important role in the defense against reactive oxygen species produced during oxidative metabolism, and contributes to DNA repair and protein synthesis (Dringen,
2000; Canugovi et al., 2013). It is important to note that trypanothione and trypanothione reductase are unique to the trypanosomatid protozoa (Vincent et al., 2016), as the main defense mecahnism against oxidative damage (Fiorillo et al., 2012). Because T. gondii lacks the genes for trypanothione biosynthesis, it is possible that trypanothione metabolites were misidentified in the present study. It is also possible that T. gondii synthesizes trypanosome-specific trypanothione metabolite using an as yet undiscovered route, but this assumption remains to be confirmed.

Infection also induced significant changes in levels of metabolites involved in amino acid metabolism (Table 2). Tryptophan, tyrosine and arginine are essential amino acids required by the parasite to sustain its own growth (Silva et al., 2002; Marino and Boothroyd, 2017). In both acutely 
TABLE 2 | List of the differentially expressed metabolites involved in amino acid metabolism during the acute and chronic phases of Toxoplasma gondii infection.

\begin{tabular}{|c|c|c|c|c|c|}
\hline Metabolites & FC & $q$-value & VIP & CV & Metabolic pathways \\
\hline \multicolumn{6}{|l|}{ ACUTELY INFECTED VS. CONTROL } \\
\hline L-Thyroxine & 0.23 & $4.73 e-03$ & 1.19 & $\downarrow$ & Tyrosine metabolism \\
\hline 3-Methoxy-4-hydroxyphenylacetaldehyde & 0.51 & $2.83 e-02$ & 1.08 & $\downarrow$ & \\
\hline gamma-Glutamyl-gamma-aminobutyraldehyde & 2.59 & $4.45 e-03$ & 1.80 & $\uparrow$ & Arginine and proline metabolism \\
\hline Nopaline & 1.84 & $6.92 \mathrm{e}-03$ & 1.21 & $\uparrow$ & \\
\hline Indole-3-ethanol & 2.17 & $5.45 e-03$ & 1.38 & $\uparrow$ & Tryptophan metabolism \\
\hline Taurocholate & 0.12 & $3.70 \mathrm{e}-02$ & 1.20 & $\downarrow$ & Taurine and hypotaurine metabolism \\
\hline Glutathionylspermidine & 1.77 & $8.84 \mathrm{e}-03$ & 1.21 & $\uparrow$ & Glutathione metabolism \\
\hline Trypanothione disulfide & 2.16 & $8.94 \mathrm{e}-03$ & 1.15 & $\uparrow$ & \\
\hline Trypanothione & 9.45 & $1.81 e-03$ & 3.28 & $\uparrow$ & \\
\hline \multicolumn{6}{|l|}{ CHRONICALLY INFECTED VS. CONTROL } \\
\hline L-Thyroxine & 0.12 & $5.00 e-04$ & 1.71 & $\downarrow$ & Tyrosine metabolism \\
\hline 3-Methoxy-4-hydroxyphenylacetaldehyde & 0.50 & $2.58 \mathrm{e}-02$ & 1.19 & $\downarrow$ & \\
\hline L-Cystathionine & 0.51 & $2.08 \mathrm{e}-02$ & 1.21 & $\downarrow$ & $\begin{array}{l}\text { Glycine, serine and threonine metabolism; Cysteine and } \\
\text { methionine metabolism }\end{array}$ \\
\hline N-Formyl-L-aspartate & 0.65 & $3.73 e-04$ & 1.03 & $\downarrow$ & Histidine metabolism \\
\hline Glutathionylspermidine & 1.58 & $3.39 \mathrm{e}-02$ & 1.01 & $\uparrow$ & Glutathione metabolism \\
\hline Trypanothione disulfide & 2.66 & $3.34 \mathrm{e}-03$ & 1.56 & $\uparrow$ & \\
\hline Trypanothione & 6.21 & $5.32 e-03$ & 2.95 & $\uparrow$ & \\
\hline
\end{tabular}

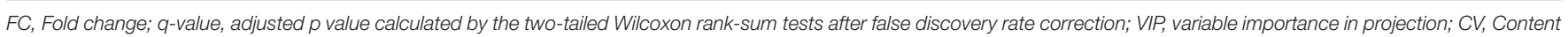
variance. A single arrow $(\uparrow / \downarrow)$ indicates increased or decreased values compared to the control group.
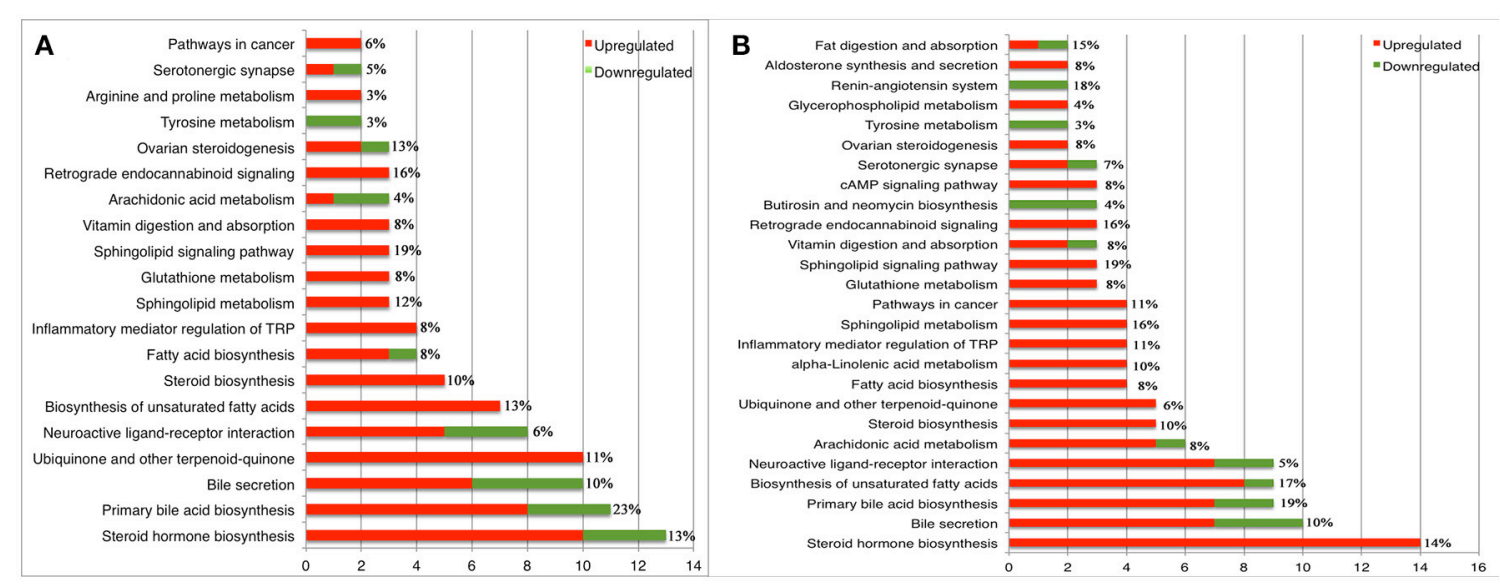

FIGURE 5 | Statistics and comparison of metabolic pathways during T. gondii infection phases. (A) Metabolic pathways of enriched metabolite $\geq 2$ of acutely infected group vs. control. (B) Metabolic pathways of enriched metabolite $\geq 2$ of chronically infected vs. control. Red and green metabolites indicate higher and lower concentrations, respectively. The $x$-axis denotes the number of differentially abundant metabolites. The $y$-axis indicates the name of metabolic pathways. The percentage represents coverage rate of the pathway, which is the differential metabolites/total metabolites in the pathway.

and chronically infected mice, L-thyroxine and 3-Methoxy-4hydroxyphenylacetaldehyde, involved in tyrosine metabolism, were downregulated. Uniquely in acutely infected mice, indole3-ethanol involved in tryptophan metabolism, and gammaglutamyl-gamma-aminobutyraldehyde and nopaline involved in arginine and proline metabolism, were upregulated. This finding is consistent with our earlier proteomic analysis of mouse liver infected with $T$. gondii, where indoleamine 2,3-dioxygenase (Ido2) was downregulated in the infected livers, probably to support the parasite growth by countering the reduction in tryptophan (He et al., 2016b). Dysregulation in the levels of these amino acids is to be anticipated because this parasite is auxotrophic for tryptophan and arginine, among other amino acids.

We previously showed, based on preotomics analysis, that T. gondii interferes with the metabolism of fatty acids, lipids and energy in the liver via the modulation of host peroxisome proliferator-activated receptor (PPAR) signaling pathway $(\mathrm{He}$ 


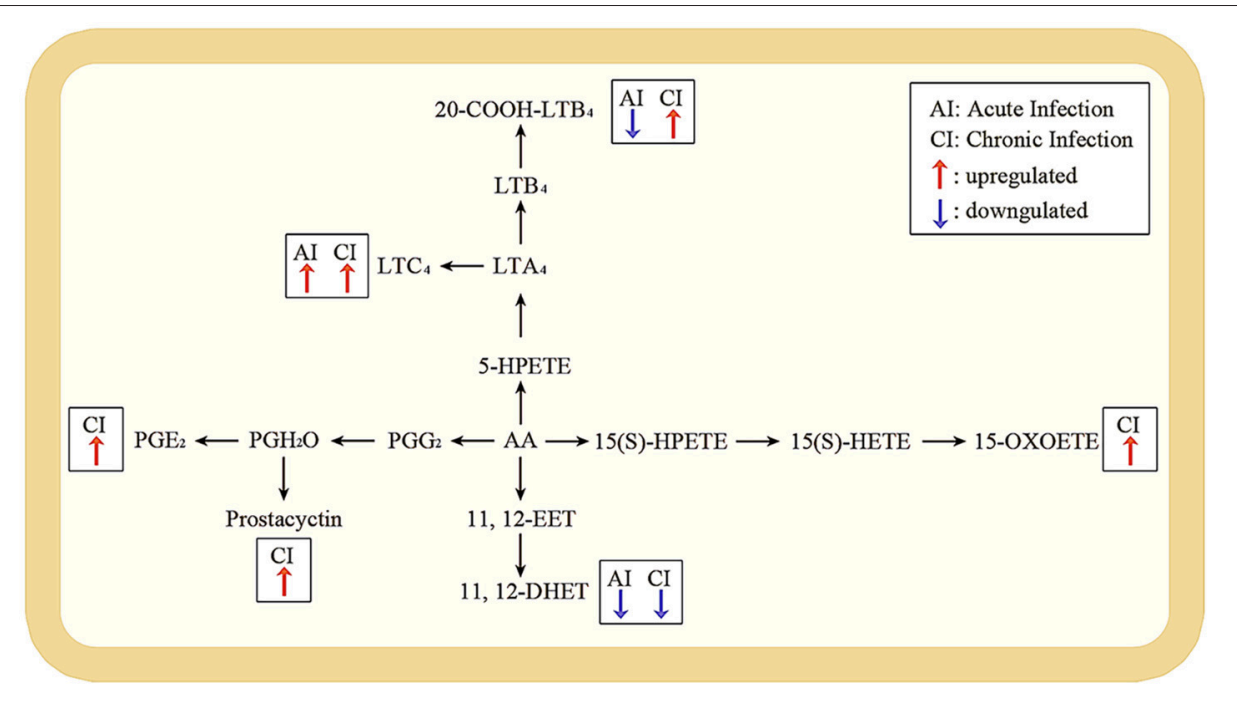

FIGURE 6 | Pathway analysis of arachidonic acid metabolism during acute and chronic infection. A schematic illustration of arachidonic acid metabolism pathway; the most significant differentially expressed metabolites during acute and chronic infection phases. Red and blue arrows represent upregulated and downregulated metabolites. The metabolites without arrows are not changed. In the acute infection, Leukotriene C4 (LTC4) was upregulated whereas 20-COOH-LB4 and 11,12-DHET were downregulated; but in the chronic infection, LTC4, 20-COOH-LB4, Prostaglandin E2 (PGE2), Prostacyctin and 15-OxoETE were upregulated with only 11,12-DHET being downregulated.

et al., 2016b). We also observed notable changes in the hepatic levels of lipids in infected mice. As shown in Figure 5 and Table 1, in acutely and chronically infected mice, the major perturbed metabolic pathways included steroid hormone biosynthesis, primary bile acid biosynthesis, steroid biosynthesis, biosynthesis of unsaturated fatty acids, fatty acid biosynthesis, and arachidonic acid metabolism. Downregulation of genes involved in bile synthesis, and the metabolism of sterol, fatty acids, and lipids have also been reported (He et al., 2016a). Steroid hormones contribute to several physiological processes; relevant to $T$. gondii infection are the immunoregulatory activities of steroids, which can influence the host immune response to infection (Tait et al., 2008). Fatty acids are essential for the synthesis of membranes of the newly produced parasites (Ramakrishnan et al., 2012) and lipids have been implicated in the pathogenesis of T. gondii infection (Milovanović et al., 2017). Upregulation of metabolites involved in sphingolipid metabolism was also observed during both phases of infection. Sphingolipids play critical roles in mediating cell-cell interaction, modulating the behavior of cellular proteins and receptors, and participate in signal transduction (Merrill, 2002).

Arachidonic acid (AA) metabolism was the most significant differentially abundant metabolic pathway between acutely and chronically infected mice. AA has been shown to promote T. gondii invasion (Li et al., 2008). The metabolism of AA was downregulated during acute infection and upregulated during chronic infection (Figure 6). In response to an inflammatory stimulus, AA, the main polyunsaturated fatty acid present in the phospholipid of cell membranes, is released and metabolized to a series of eicosanoids, including the inflammatory leukotrienes and prostaglandins (Greene et al., 2011). AA and its inflammatory metabolites mediate the regulation of key cellular processes, such as cell survival, angiogenesis, chemotaxis, mitogenesis, apoptosis and migration (Rizzo et al., 1999; Levick et al., 2007). Alpha-linolenic acid (ALA), an essential omega-3 fatty acid that is commonly found in seeds, nuts, and vegetable oils, was perturbed during chronic stage of infection. However, because ALA is an essential fatty acid (i.e., cannot be synthesized by the mice), its increased level must have been derived from the mouse diet.

Our results showed a stronger impact of the parasite infection on the liver metabolic status during acute compared to chronic infection. However, one possible reason for the metabolic alterations observed during chronic infection may be partly related to considering $30 \mathrm{dpi}$ as the chronic phase of T. gondii infection, which is a relatively early time point to mark the latency of $T$. gondii infection. In fact, a previous study showed low parasite burden and absence of parasite DNA in the liver of Swiss albino mice infected with T. gondii type II Me49 strain at 28 and 42 dpi, respectively (Djurković-Djaković et al., 2012).

\section{CONCLUSION}

By taking a global approach to understanding the metabolomics of mouse liver in the context of $T$. gondii infection, we have identified a number of previously known and novel metabolites that play biological roles relevant to host-parasite interaction. Our data indicate that mouse liver harbors intrinsic mechanisms to restrict T. gondii replication. For example, through the upregulation of GSH metabolism, liver can buffer oxidative stress associated with infection. On the other hand, the parasite has evolved mechanisms to exploit various lipids and amino acids, to support its own proliferation. Our metabolic profiling also 
showed many altered metabolites and metabolic pathways (e.g., steroid hormone biosynthesis, primary bile acid biosynthesis, bile secretion, and biosynthesis of unsaturated fatty acids) in the liver of T. gondii-infected mice during acute and chronic infections when compared with healthy uninfected mice. These findings provide new insight into metabolic changes that occur in the liver in acute and chronic murine toxoplasmosis.

\section{DATA AVAILABILITY STATEMENT}

The datasets supporting the findings of this article are included within the article. The metabolomics data are available in the MetaboLights database (MTBLS586) (http://www.ebi.ac.uk/ metabolights/).

\section{AUTHOR CONTRIBUTIONS}

$\mathrm{X}-\mathrm{QZ}$ and C-XZ conceived and designed the experiments. XQC and C-XZ performed the experiments. X-QC analyzed the data and wrote the paper. R-SH, G-XH, and S-LG helped in the analyzing of the data. X-QC, HE and X-QZ critically revised the manuscript. All authors read and approved the final version of the manuscript.

\section{FUNDING}

Project support was provided by the National Natural Science Foundation of China (Grant No. 31230073), by the International Science and Technology Cooperation Project of Gansu Province (Grant No. 17JR7WA031), by the Elite Program of Chinese Academy of Agricultural Sciences, by the Agricultural Science and Technology Innovation Program (ASTIP) (Grant No. CAAS-ASTIP-2016-LVRI-03), and by the Basal Research Fund of Shandong University (Grant No. 21510077614097).

\section{ACKNOWLEDGMENTS}

We thank BGI-Shenzhen for technical assistance with the LC-MS/MS analysis. We would also like to thank Dr. Mohamed Metwally from Zagazig University for helping with histopathological analysis and the reviewers for very constructive, fair and objective comments.

\section{REFERENCES}

Antunes, L. C., Arena, E. T., Menendez, A., Han, J., Ferreira, R. B., Buckner, M. M., et al. (2011). Impact of Salmonella infection on host hormone metabolism revealed by metabolomics. Infect. Immun. 79, 1759-1769. doi: 10.1128/IAI.01373-10

Canugovi, C., Misiak, M., Ferrarelli, L. K., Croteau, D. L., and Bohr, V. A. (2013). The role of DNA repair in brain related disease pathology. DNA repair (Amst) 12, 578-587. doi: 10.1016/j.dnarep.2013.04.010

Charron, A. J., and Sibley, L. D. (2002). Host cells: mobilizable lipid resources for the intracellular parasite Toxoplasma gondii. J. Cell Sci. 115, 3049-3059.

Chaudhary, K., Darling, J. A., Fohl, L. M., Sullivan, W. J. Jr., Donald, R. G., Pfefferkorn, E. R., et al. (2004). Purine salvage pathways in the

\section{SUPPLEMENTARY MATERIAL}

The Supplementary Material for this article can be found online at: https://www.frontiersin.org/articles/10.3389/fcimb. 2018.00189/full\#supplementary-material

Figure $\mathbf{S 1}$ | The original image of Figure $\mathbf{1 A}$.

Figure $\mathbf{S 2}$ | The original image of Figure 2B.

Figure $\mathbf{S} \mathbf{3}$ | The original image of Figure $\mathbf{3 C}$.

Figure S4 (A) | Total ion current (TIC) chromatograms of liver samples in the positive ion mode (ESI+). (B) Total ion current (TIC) chromatograms of liver samples in the negative ion mode (ESI-).

Figure S5 | Secondary spectrogram information of anandamide. (A) Standard secondary spectrogram of anandamide in the positive ion mode (ESI+). (B) Secondary spectrogram of experimental samples at similar retention time in the positive ion mode (ESI+).

Figure S6 | Secondary spectrogram information of cholesterol. (A) Standard secondary spectrogram of cholesterol in the positive ion mode (ESI+). (B) Secondary spectrogram of experimental samples at similar retention time in the positive ion mode $(\mathrm{ESI}+)$.

Figure S7 | Secondary spectrogram information of choli acid. (A) Standard secondary spectrogram of choli acid in the positive ion mode (ESI+). (B) Secondary spectrogram of experimental samples at similar retention time in the positive ion mode $(\mathrm{ESI}+)$.

Figure S8 | Secondary spectrogram information of lithocholic acid. (A) Standard secondary spectrogram of lithocholic acid in the positive ion mode (ESI+). (B) Secondary spectrogram of experimental samples at similar retention time in the positive ion mode $(\mathrm{ESI}+)$.

Figure S9 | Secondary spectrogram information of L-Thyroxine. (A) Standard secondary spectrogram of L-Thyroxine in the positive ion mode (ESI+). (B) Secondary spectrogram of experimental samples at similar retention time in the positive ion mode $(E S I+)$.

Figure S10 | Secondary spectrogram information of oleic acid. (A) Standard secondary spectrogram of oleic acid in the positive ion mode (ESI+). (B) Secondary spectrogram of experimental samples at similar retention time in the positive ion mode $(E S I+)$.

Figure S11 | Secondary spectrogram information of sphinganine. (A) Standard secondary spectrogram of sphinganine in the positive ion mode (ESI+). (B) Secondary spectrogram of experimental samples at similar retention time in the positive ion mode (ESI+).

Table S1 | Summary results of the differential ions.

Table S2 | List of metabolites identified during acute Toxoplasma gondii infection.

Table S3 | List of metabolites identified during chronic Toxoplasma gondii infection.

apicomplexan parasite Toxoplasma gondii. J. Biol. Chem. 279, 31221-31227. doi: $10.1074 /$ jbc.M404232200

Chen, X. Q., Zhou, C. X., Elsheikha, H. M., He, S., Hu, G. X., and Zhu, X. Q. (2017). Profiling of the perturbed metabolomic state of mouse spleen during acute and chronic toxoplasmosis. Parasite Vectors 10:339. doi: 10.1186/s13071-017-2282-6

Cong, W., Zhang, X. X., He, J. J., Li, F. C., Elsheikha, H. M., and Zhu, X. Q. (2017). Global miRNA expression profiling of domestic cat livers following acute Toxoplasma gondii infection. Oncotarget 8, 25599-25611. doi: 10.18632 /oncotarget.16108

Coppens, I., and Joiner, K. A. (2003). Host but not parasite cholesterol controls Toxoplasma cell entry by modulating organelle discharge. Mol. Biol. Cell 14, 3804-3820. doi: 10.1091/mbc.e02-12-0830 
Coppens, I., Sinai, A. P., and Joiner, K. A. (2000). Toxoplasma gondii exploits host low-density lipoprotein receptor- mediated endocytosis for cholesterol acquisition. J. Cell Biol. 149, 167-180. doi: 10.1083/jcb.149.1.167

Dai, W., Pan, H., Kwok, O., and Dubey, J. P. (1994). Human indoleamine 2,3dioxygenase inhibits Toxoplasma gondii growth in fibroblast cells. J. Interferon Res. 14, 313-317. doi: 10.1089/jir.1994.14.313

Dimier, I. H., and Bout, D. T. (1998). Interferon-gamma-activated primary enterocytes inhibit Toxoplasma gondii replication: a role for intracellular iron. Immunol. 94, 488-495. doi: 10.1046/j.1365-2567.1998.00553.x

Djurković-Djaković, O., Djokić, V., Vujanić, M., Zivković, T., Bobić, B., Nikolić, A., et al. (2012). Kinetics of parasite burdens in blood and tissues during murine toxoplasmosis. Exp. Parasitol. 131, 372-376. doi: 10.1016/j.exppara.2012.05.006

Dringen, R. (2000). Metabolism and functions of glutathione in brain. Prog Neurobiol. 62, 649-671. doi: 10.1016/S0301-0082(99)00060-X

Dubey, J. P. (2008). The history of Toxoplasma gondii-the first 100 years. J. Eukaryot. Microbiol. 55, 467-475. doi: 10.1111/j.1550-7408.2008.00345.x

Dubey, J. P., and Beattie, C. P. (1988). Toxoplasmosis of Animals and Man. Boca Raton, FL: CRC Press.

Dubey, J. P., and Frenkel, J. K. (1976). Feline toxoplasmosis from acutely infected mice and the development of Toxoplasma cysts. J. Protozool. 23, 537-546. doi: 10.1111/j.1550-7408.1976.tb03836.x

Dupont, C. D., Christian, D. A., and Hunter, C. A. (2012). Immune response and immunopathology during toxoplasmosis. Semin. Immunopathol. 34, 793-813. doi: 10.1007/s00281-012-0339-3

Elsheikha, H. M. (2008). Congenital toxoplasmosis: priorities for further health promotion action. Public Health 122, 335-353. doi: 10.1016/j.puhe.2007.08.009

Elsheikha, H. M., and Zhu, X. Q. (2016). Toxoplasma gondii infection and schizophrenia: an inter-kingdom communication perspective. Curr. Opin. Infect. Dis. 29, 311-318. doi: 10.1097/QCO.0000000000000265

Fiorillo, A., Colotti, G., Boffi, A., Baiocco, P., and Ilari, A. (2012). The crystal structures of the tryparedoxin-tryparedoxin peroxidase couple unveil the structural determinants of Leishmania detoxification pathway. PLoS Negl. Trop. Dis. 6:e1781. doi: 10.1371/journal.pntd.0001781

Fox, B. A., Gigley, J. P., and Bzik, D. J. (2004). Toxoplasma gondii lacks the enzymes required for de novo arginine biosynthesis and arginine starvation triggers cyst formation. Int. J. Parasitol. 34, 323-331. doi: 10.1016/j.ijpara.2003.12.001

Greene, E. R., Huang, S., Serhan, C. N., and Panigrahy, D. (2011). Regulation of inflammation in cancer by eicosanoids. Prostaglandins Other Lipid Mediat. 96, 27-36. doi: 10.1016/j.prostaglandins.2011.08.004

He, J. J., Ma, J., Elsheikha, H. M., Song, H. Q., Zhou, D. H., and Zhu, X. Q. (2016b). Proteomic profiling of Mouse liver following acute Toxoplasma gondii infection. PLoS ONE 11:e0152022. doi: 10.1371/journal.pone.0152022

He, J. J., Ma, J., Elsheikha, H. M., Song, H. Q., and Zhu, X. Q. (2016a). Transcriptomic analysis of mouse liver reveals a potential hepato-enteric pathogenic mechanism in acute Toxoplasma gondii infection. Parasit. Vectors 9:427. doi: 10.1186/s13071-016-1716-X

Hill, D. E., Chirukandoth, S., Dubey, J. P., Lunney, J. K., and Gamble, H. R. (2006). Comparison of detection methods for Toxoplasma gondii in naturally and experimentally infected swine. Vet. Parasitol. 141, 9-17. doi: 10.1016/j.vetpar.2006.05.008

Holmes, E., Wilson, I. D., and Nicholson, J. K. (2008). Metabolic phenotyping in health and disease. Cell 134, 714-717. doi: 10.1016/j.cell.2008.08.026

Kafsack, B. F., and Llinás, M. (2010). Eating at the table of another: metabolomics of host-parasite interactions. Cell Host Microbe. 7, 90-99. doi: 10.1016/j.chom.2010.01.008

Levick, S. P., Loch, D. C., Taylor, S. M., and Janicki, J. S. (2007). Arachidonic acid metabolism as a potential mediator of cardiac fibrosis associated with inflammation. J. Immunol. 178, 641-646. doi: 10.4049/jimmunol.178.2.641

Li, L., Li, X., and Yan, J. (2008). Alterations of concentrations of calcium and arachidonic acid and agglutinations of microfilaments in host cells during Toxoplasma gondii invasion. Vet. Parasitol. 157, 21-23. doi: 10.1016/j.vetpar.2008.07.007

Marino, N. D., and Boothroyd, J. C. (2017). Toxoplasma growth in vitro is dependent on exogenous tyrosine and is independent of AAH2 even in tyrosine-limiting conditions. Exp. Parasitol. 176, 52-58. doi: $10.1016 /$ j.exppara.2017.02.018
Merrill, A. H. Jr. (2002). De novo sphingolipid biosynthesis: a necessary, but dangerous, pathway. J. Biol. Chem. 277, 25843-25846. doi: 10.1074/jbc.R200009200

Milovanović, I., Busarcević, M., Trbovich, A., Ivović, V., and Uzelac, A. (2017). Evidence for host genetic regulation of altered lipid metabolism in experimental toxoplasmosis supported with gene data mining results. PLoS ONE 12:e0176700. doi: 10.1371/journal.pone.0176700

Munoz, M., Liesenfeld, O., and Heimesaat, M. M. (2011). Immunology of Toxoplasma gondii. Immunol. Rev. 240, 269-285. doi: 10.1111/j.1600-065X.2010.00992.x

Naemat, A., Elsheikha, H. M., Boitor, R. A., and Notingher, I. (2016). Tracing amino acid exchange during host-pathogen interaction by combined stable-isotope time-resolved Raman spectral imaging. Sci. Rep. 6:20811. doi: 10.1038/srep20811

Nelson, M. M., Jones, A. R., Carmen, J. C., Sinai, A. P., Burchmore, R., and Wastling, J. M. (2008). Modulation of the host cell proteome by the intracellular apicomplexan parasite Toxoplasma gondii. Infect. Immun. 76, 828-844. doi: 10.1128/IAI.01115-07

Nguyen, C. T., Shetty, V., and Maresso, A. W. (2015). Global metabolomic analysis of a mammalian host infected with Bacillus anthracis. Infect. Immun. 83, 4811-4825. doi: 10.1128/IAI.00947-15

Nicholson, J. K., Lindon, J. C., and Holmes, E. (1999). 'Metabonomics': understanding the metabolic responses of living systems to pathophysiological stimuli via multivariate statistical analysis of biological NMR spectroscopic data. Xenobiotica 29, 1181-1189.

Pfefferkorn, E. R. (1984). Interferon gamma blocks the growth of Toxoplasma gondii in human fibroblasts by inducing the host cells to degrade tryptophan. Proc. Natl. Acad. Sci. U.S.A. 81, 908-912. doi: 10.1073/pnas.81.3.908

Pittman, K. J., Aliota, M. T., and Knoll, L. J. (2014). Dual transcriptional profiling of mice and Toxoplasma gondii during acute and chronic infection. BMC Genomics 15:806. doi: 10.1186/1471-2164-15-806

Ramakrishnan, S., Docampo, M. D., Macrae, J. I., Pujol, F. M., Brooks, C. F., van Dooren, G. G., et al. (2012). Apicoplast and endoplasmic reticulum cooperate in fatty acid biosynthesis in apicomplexan parasite Toxoplasma gondii. J. Biol. Chem. 287, 4957-4971. doi: 10.1074/jbc.M111.310144

Rizzo, M. T., Regazzi, E., Garau, D., Akard, L., Dugan, M., Boswell, H. S., et al. (1999). Induction of apoptosis by arachidonic acid in chronic myeloid leukemia cells. Cancer Res. 59, 5047-5053.

Rosa, F. T., Freitas, E. C., Deminice, R., Jordão, A. A., and Marchini, J. S. (2014). Oxidative stress and inflammation in obesity after taurine supplementation: a double-blind, placebo-controlled study. Eur. J. Nutr. 53, 823-830. doi: 10.1007/s00394-013-0586-7

Schwartzman, J. D., and Pfefferkorn, E. R. (1982). Toxoplasma gondii: purine synthesis and salvage in mutant host cells and parasites. Exp. Parasitol. 53, 77-86. doi: 10.1016/0014-4894(82)90094-7

Seabra, S. H., DaMatta, R. A., de Mello, F. G., and de Souza, W. (2004). Endogenous polyamine levels in macrophages is sufficient to support growth of Toxoplasma gondii. J. Parasitol. 90, 455-460. doi: 10.1645/GE-179R

Shin, J. H., Yang, J. Y., Jeon, B. Y., Yoon, Y. J., Cho, S. N., Kang, Y. H., et al. (2011). $1 \mathrm{H}$ NMR-based metabolomic profiling in mice infected with Mycobacterium tuberculosis. J. Proteome Res. 10, 2238-2247. doi: 10.1021/pr101054m

Silva, N. M., Rodrigues, C. V., and Santoro, M. M. (2002). Expression of indoleamine 2,3-dioxygenase, tryptophan degradation, and kynurenine formation during in vivo infection with Toxoplasma gondii: induction by endogenous gamma interferon and requirement of interferon regulatory factor 1. Infect. Immun. 70, 859-868. doi: 10.1128/IAI.70.2.859-868.2002

Sullivan, W. J. Jr., and Jeffers, V. (2012). Mechanisms of Toxoplasma gondii persistence and latency. FEMS Microbiol. Rev. 36, 717-733. doi: 10.1111/j.1574-6976.2011.00305.x

Tait, A. S., Butts, C. L., and Sternberg, E. M. (2008). The role of glucocorticoids and progestins in inflammatory, autoimmune, and infectious disease. J. Leukoc. Biol. 84, 924-931. doi: 10.1189/jlb.0208104

Vincent, I. M., Daly, R., Courtioux, B., Cattanach, A. M., Biéler, S., Ndung’u, J. M., et al. (2016). Metabolomics identifies multiple candidate biomarkers to diagnose and stage human african trypanosomiasis. PLoS Negl. Trop. Dis. 10:e0005140. doi: 10.1371/journal.pntd.0005140 
Vischer, T. L., Bernheim, C., and Engelbrecht, E. (1967). Two cases of hepatitis due to Toxoplasma gondii. Lancet 290, 919-921. doi: 10.1016/S0140-6736(67)90235-8

Weitberg, A. B., Alper, J. C., Diamond, I., and Fligiel, Z. (1979). Acute granulomatous hepatitis in the course of acquired toxoplasmosis. N. Engl. J. Med. 300, 1093-1096. doi: 10.1056/NEJM197905103001907

Wen, B., Mei, Z., Zeng, C., and Liu, S. (2017). metaX: a flexible and comprehensive software for processing metabolomics data. BMC Bioinformatics 18:183. doi: 10.1186/s12859-017-1579-y

Wendum, D., Carbonell, N., Svrcek, M., Chazouilléres, O., and Fléjou, J. (2002). Fatal disseminated toxoplasmosis in a toxoplasma seropositive liver transplant recipient. J. Clin. Pathol. 55:637. doi: 10.1136/jcp.55.8.637

Yang, J., Zhao, X., Liu, X., Wang, C., Gao, P., Wang, J., et al. (2006). High performance liquid chromatography-mass spectrometry for metabonomics: potential biomarkers for acute deterioration of liver function in chronic hepatitis B. J. Proteome Res. 5, 554-561. doi: 10.1021/pr050364w

Zhong, H., Fang, C., Fan, Y., Lu, Y., Wen, B., Ren, H., et al. (2017). Lipidomic profiling reveals distinct differences in plasma lipid composition in healthy, prediabetic and type 2 diabetic individuals. Gigascience 6, 1-12. doi: 10.1093/gigascience/gix036
Zhou, C. X., Zhou, D. H., Elsheikha, H. M., Liu, G. X., Suo, X., and Zhu, X. Q. (2015). Global metabolomic profiling of mice brains following experimental infection with the cyst-forming Toxoplasma gondii. PLoS ONE 10:e139635. doi: 10.1371/journal.pone.0139635

Zhou, C. X., Zhou, D. H., Elsheikha, H. M., Zhao, Y., Suo, X., and Zhu, X. Q. (2016). Metabolomic profiling of mice serum during toxoplasmosis progression using liquid chromatography-mass spectrometry. Sci. Rep. 6:19557. doi: $10.1038 /$ srep 19557

Conflict of Interest Statement: The authors declare that the research was conducted in the absence of any commercial or financial relationships that could be construed as a potential conflict of interest.

Copyright (c) 2018 Chen, Elsheikha, Hu, Hu, Guo, Zhou and Zhu. This is an openaccess article distributed under the terms of the Creative Commons Attribution License (CC BY). The use, distribution or reproduction in other forums is permitted, provided the original author(s) and the copyright owner are credited and that the original publication in this journal is cited, in accordance with accepted academic practice. No use, distribution or reproduction is permitted which does not comply with these terms. 